\title{
CAE Model Correlation \& Design Optimization of a Laminated Steel Oil Pan by means of Acceleration and Strain Measurement on a Fired Engine
}

\author{
Rıfat K. Yanarocak*», Abdulkadir Çekiç ** \\ *Ford Otomotiv San. A.S., Department of Design Verification \& Test, Design Verification \& Test Engineer, \\ Akpınar Mah., Hasan Basri Cad, No: 2, PK: 34885 Sancaktepe/ ISTANBUL/TURKEY \\ ** Ford Otomotiv San. A.S., Department of Powertrain CAE, Structural Analysis Engineer, \\ Akpınar Mah., Hasan Basri Cad, No: 2, PK: 34885 Sancaktepe/ ISTANBUL/TURKEY \\ ryanaroc@ford.com, acekic1@ford.com \\ ${ }^{\ddagger}$ Corresponding Author: Rifat K. YANAROCAK, Akpınar Mah., Hasan Basri Cad, No: 2, PK: 34885 Sancaktepe/ \\ ISTANBUL/TURKEY, Tel: +90 216664 9305, Fax: +90 216664 9305, ryanaroc@ ford.com
}

Received: 16.01.2015 Accepted: 06.03.2015

\begin{abstract}
In this paper, a simultaneous design and development work for a diesel engine oil pan is presented. The interesting point making the design of the oil pan so special is its laminated steel material. Beside its material, due to the deep-drawing production method, to determine the natural frequencies of the structure with Computer Aided Engineering (CAE) methodology is a problem for the design engineer. Especially when the highly nonlinear character of the oil pan and regions at different thicknesses sum up with the hardness of liquid modelling, free vibration modal analysis of the design at virtual environment becomes extremely challenging. Therefore, instead of refining the material characteristics in virtual design, first a primary 3D dummy design is generated. Afterwards, a production method and material intent sample is produced with soft tool. Hammer test is applied on this sample filled with oil, therefore modal shapes and frequencies are gained. As a result, CAE modal analysis is generated and correlated by hammer test results; hence the first challenge of modelling the liquid is overcome. Then, critical stress locations are determined with the CAE durability analysis. After instrumenting the pan with optimum number of accelerometers and strain-gages from these critical locations, a durability test on a fired engine is run. With the measurements here, the CAE durability analysis is refined so the second challenge of material nonlinearity and thinning due to the deep-drawing method is also overcome. Lastly, with the CAE durability simulation, a secondary 3D design proposal is established. Normally the method reach to success here, but to validate it, a secondary sample is produced, instrumented again with accelerometers \& gages and tested resulting in significant improvement in terms of durability. With this approach, a method to perform a fail-safe oil pan design in single loop is verified.
\end{abstract}

Keywords Oil pan, laminated steel, deep drawing, CAE durability analysis, advanced instrumentation.

\section{Introduction}

Oil pan is a reservoir usually located at the base of the engine cylinder block enclosing the crankcase. It is manufactured from number of different materials like cast aluminium, stamped mild steel, laminated steels and various plastics \& synthetic fibers. [3] Besides transferring lube oil heat to atmosphere, oil pans, can also act as a sort of boom box and amplify engine noise, so they are usually designed to minimize noise and vibration. [4] The effectiveness of these roles obviously has a lot to do with determination of the right material from which the oil pan is manufactured. As a result in the new generation diesel engines, due to its effective noise reducing and durability improving properties laminated steel is used commonly.

Beside its material, the production method of the oil pan is also an important aspect determining its durability. At this point, the deep drawn part manufacturing technology which 
offers a number of benefits over other manufacturing processes is used. It enables the design engineer to produce large, seamless parts with complex axi-symmetric geometries in rapid cycle times and reduce technical labour. [8] But despite of all these advantages, the laminated MPM (metalplastic-metal) material, combined with the nonhomogeneous thickness problem due to deep drawing, cause the oil pan to show highly nonlinear character during free vibration modal analysis at virtual environment. [6] Especially modelling the unstable liquid inside the pan, determining the damping ratios and gaining modal shapes and frequencies of the structure only with virtual environment is extremely challenging for the design engineer.

Due to all these challenges, instead of trying to refine the material characteristics, optimize the model and create different design recommendations in virtual design, a simultaneous design and development study that would integrate experimental measurements with the CAE durability analysis would be more realistic, accurate and less time consuming. Using this methodology it is also possible to develop a fail-safe oil pan design in single loop.

\section{Experiment}

\subsection{Testing Methodology}

For the simultaneous design \& development and optimization study of the oil pan first of all a methodology consists of a fail-safe single loop is generated. The detailed steps of this methodology are listed at Table 1.

Hammer test is applied on this sample filled with oil, therefore modal shapes and frequencies are gained. As a result, CAE modal analysis is generated and correlated by hammer test results. Hence, the first challenge of modelling the liquid is overcome. In Figure 1, the application of the hammer test can be observed. Also, similar CAE model and hammer test frequency results comparison at four different modes are given.

Table 1. Single Loop Design Methodology

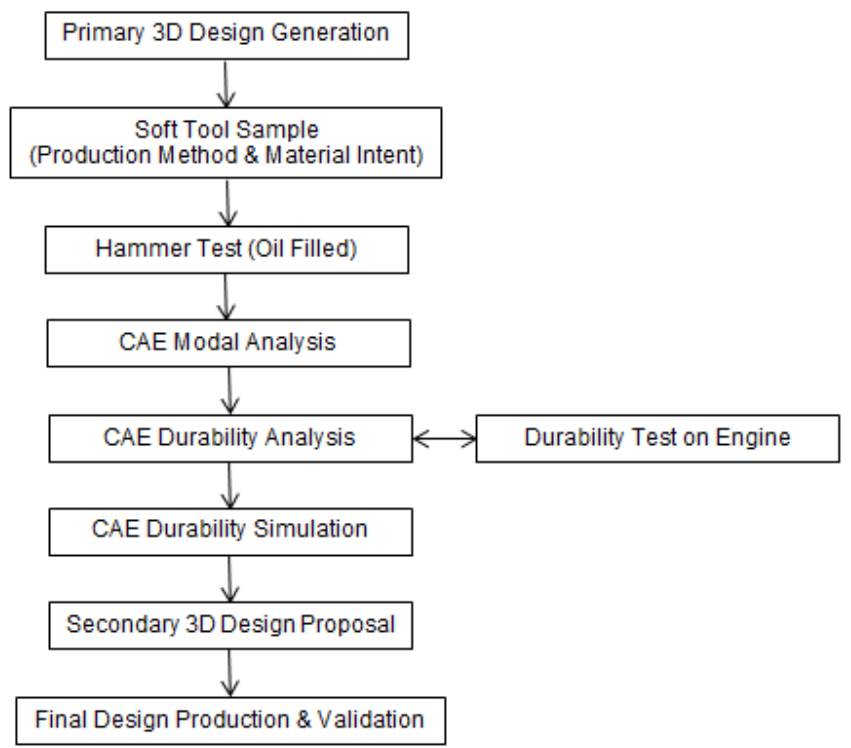

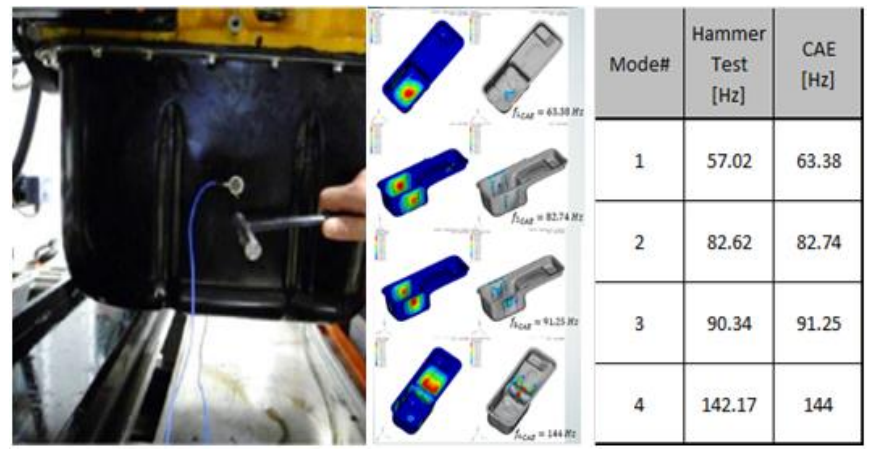

Fig. 1. Hammer Test on Oil Pan

Then, critical stress locations, magnitudes, and directions are determined with the CAE durability analysis. This is both important in terms of determining the number and type of the measurement devices. After instrumenting the oil pan with optimum number of accelerometers and strain-gages from these critical locations, a durability test on a fired engine is run. The importance of this test is the engine's being tested until failure, so it is possible to observe and compare the critical stress locations with the model

Just before the durability test the engine was tested with two different combinations of oil filling amount which has a direct effect to the natural frequency of the oil pan. During this test a power curve is taken at full load to obtain the acceleration values on the oil pan at every $100 \mathrm{rpm}$ increments. Additionally a speed sweep is performed to get strain values at high frequency between the boundary speed limits of the engine. Therefore, this helps the design engineer to determine and compare the critical stress locations on the oil pan with the frequency response analysis by using finite element analysis methods and correlate the dynamic properties of the model accordingly. Another achievement is to eliminate the effect of the level of oil to a possible damage or failure mode on the oil pan. With the measurements here, the CAE durability analysis is refined so the second challenge of material nonlinearity and thinning due to the deep-drawing method is also overcome.

Lastly, with the CAE durability simulation and model refinement, a secondary more robust $3 \mathrm{D}$ design proposal is established. A secondary sample is produced, instrumented again with accelerometers \& gages. The tests are repeated again from the most critical stress points and significant improvement is gained in terms of vibration characteristics. Also, critical stress locations are moved from side walls of the oil pan to the very bottom of the oil pan side walls with much lower magnitudes and are unlikely to damage the sump. As a result, this methodology is validated.

\subsection{Test Sample Preparation}

Experiments were performed on the oil pan of a diesel engine. In Figure 2 a similar oil pan design is shown. 


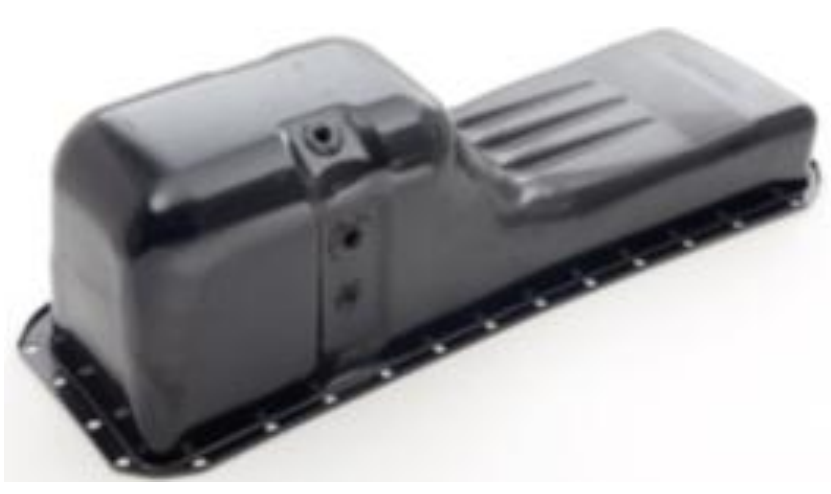

Fig. 2. Similar Oil Pan Design

Four pieces of tri-axial accelerometers and four pieces of rectangular rosette type strain-gages are instrumented very near to the critical stress regions on the left, right, front and bottom of the oil pan. Also four pieces of tri-axial accelerometers are instrumented on the right, front, front-left and rear-left of the block to separate the vibration characteristics of the oil pan from the rest of the engine. In Figure 3 the accelerometer and strain gage instrumentation on the bottom of the oil pan can be observed.

The instrumentations are performed near to the critical stress locations on the oil pan where the vibration characteristics are more stable, therefore, a better CAE model correlation is aimed. Also, special care is applied for the sensors not to be damaged during the instrumentation of the oil pan to the engine. After all the instrumentation and oil filling operations, nulling is applied to the sensors so only the effect of vibration during engine operation is gained. In Figure 4 the exact strain gage and accelerometer locations are shown.

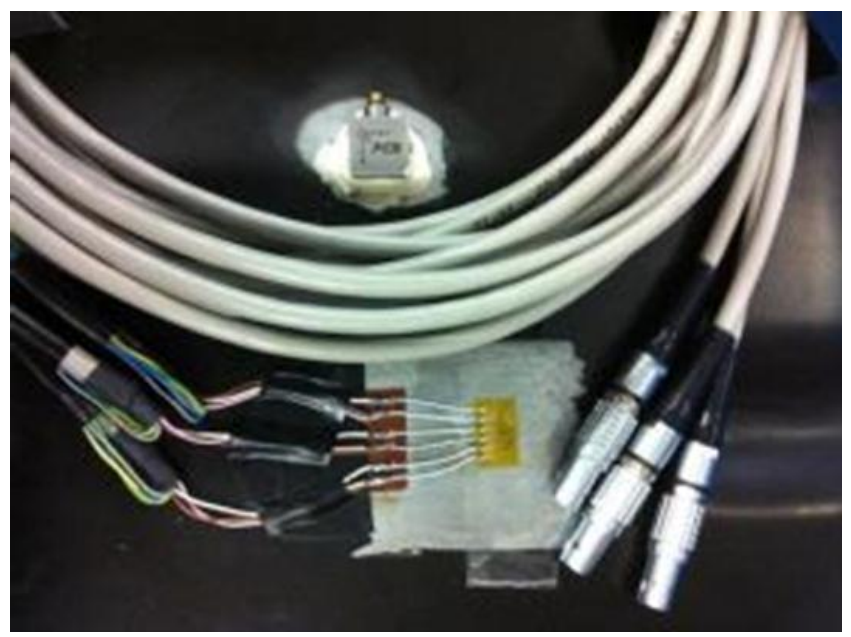

Fig. 3. Accelerometer \& Strain-gage Instrumentation on the Oil Pan

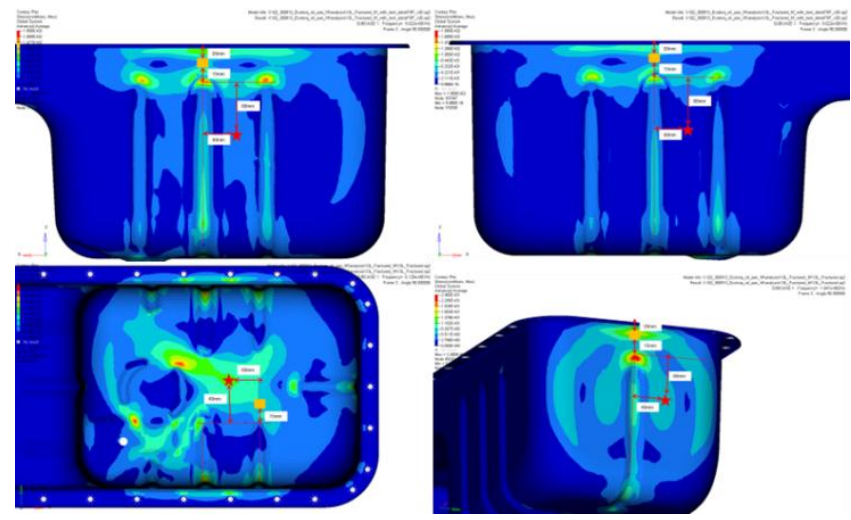

Fig. 4. Exact Accelerometer \& Strain-gage Locations on the Oil Pan

\section{Experimental Results}

Acceleration data from 4 accelerometers at the block and 4 accelerometers at the oil pan is taken by $1 \mathrm{~Hz}$ increments with $100 \mathrm{rpm}$ steps from $2100 \mathrm{rpm}$ to $600 \mathrm{rpm}$ at the power curve. The amplitude - phase and real - imaginaire values are obtained for all X, Y and $\mathrm{Z}$ directions. In Figure 5 the test cycle is shown.

The data is observed until $600 \mathrm{~Hz}$ and $1800 \mathrm{rpm}$ found to be most critical for $30 \mathrm{~L}$ of oil filling according to the acceleration values at $\mathrm{Y}$ direction of oil pan where as for $50 \mathrm{~L}$ of oil filling $1300 \mathrm{rpm}$ found to be most critical reaching more than 2 times than the values gained in 30L of oil filling, again at $\mathrm{Y}$ direction of left side of oil pan.

Strain data from 4 strain gages at the oil pan is taken by $1 \mathrm{~Hz}$ increments with $1 \mathrm{rpm}$ steps from $600 \mathrm{rpm}$ to $2100 \mathrm{rpm}$ at the speed sweeps. First of all, to eliminate the effects of static and thermal loads a drift offset correction is applied from the data taken. In Figure 6 this successful drifting operation can be observed. The below blue curve shows corrected data.

All max. \& min. principle strain \& stress values and Von Mises stress values are obtained from the data for all 4 different strain gage locations. By taking the Young Modulus as $210000 \mathrm{MPa}$ and the Poisson ratio as 0,28 again the $\mathrm{Y}$ direction of left side of the oil pan found to be the most critical stress location. Max. principle stress value is found to be almost 6 times at 50L oil filling compared to 30L.

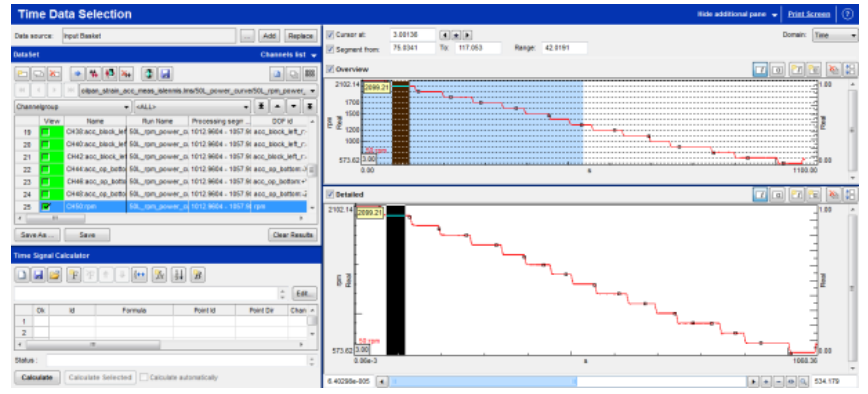

Fig. 5. Test Cycle \& Determination of the Acceleration Values 


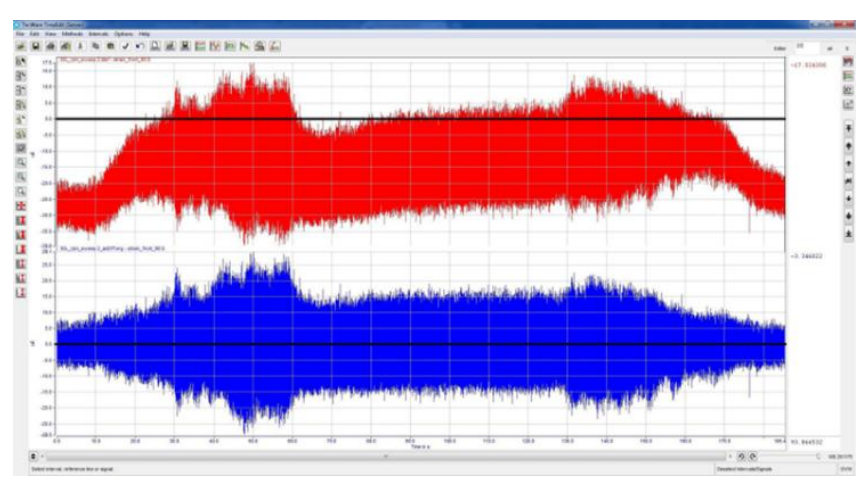

Fig. 6. Drift Offset Correction on the Strain Data

\section{CAE Methodology}

In this part, brief explanation of CAE model is given. Also, CAE correlation study by using test data is explained and analysis results are shown.

\subsection{FEA Model}

Oil pan is made of laminated steel and operates with oil inside of it. Difficult part of modelling the oil pan is to model a material which has two layers of steel and between two layers, there is a material that improve damping characteristic of oil pan.(Figure 7)

MSC NASTRAN is used to solve problem. Oil pan is modelled with 2D elements (CQUAD) and oil pan to engine block connection is modelled with bush elements (CBUSH). The effect of oil inside the oil pan is modelled by adding non-structural mass to $2 \mathrm{D}$ elements. [9] Figure 8 shows the mesh example of the oil pan.

RKU method, named after its developers Ross, Kerwin and Ungar, is the most commonly used method for characterizing the behaviour of three-layer damping treatments [1]. This method uses a fourth order differential equation for a uniform beam with the sandwich construction of the 3-layer laminate system represented as an equivalent complex stiffness. The advantages of this method are,

- No need to re-mesh if any thickness of layer in laminated steel is changed.

- It has faster run times.

- Only need to change PSHELL \& MAT1 cards

By adding bending rigidity into MAT1 card in NASTRAN, material property is modelled.

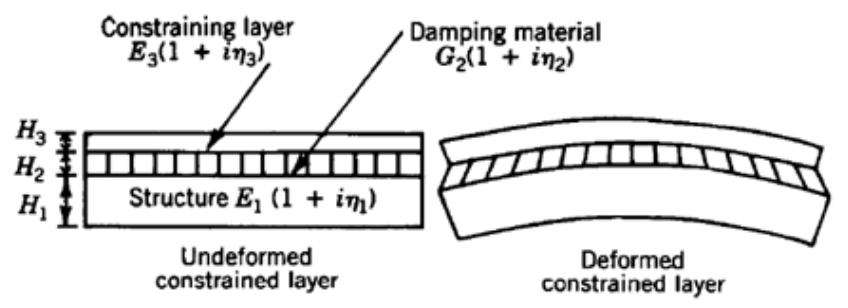

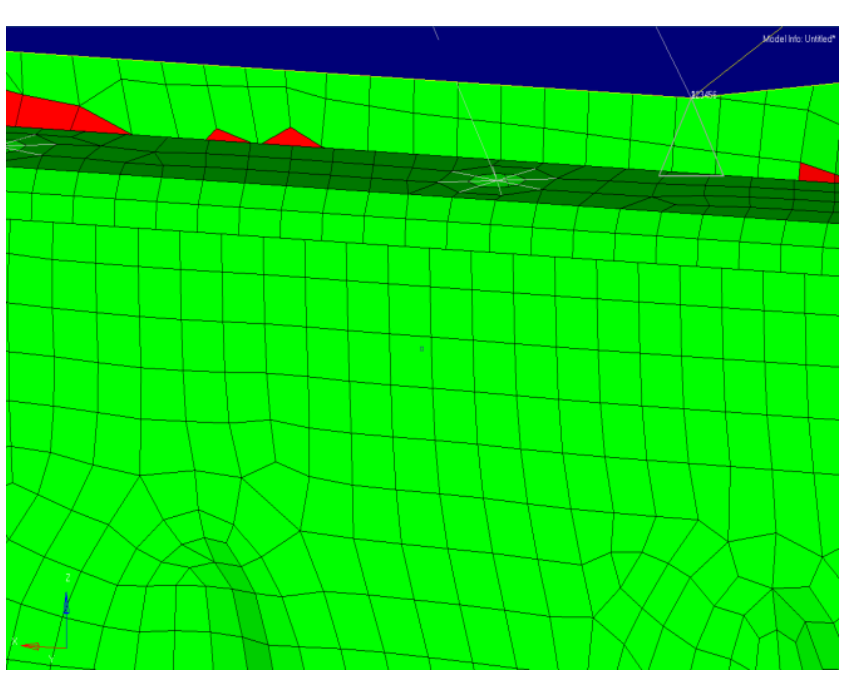

Fig. 8. Mesh Example of Oil Pan

\subsection{FEA Correlation}

Aim of the correlation is to optimize the design by using the test data. To achieve this, the main steps that are mentioned in Figure 9 are followed. Firstly, critical regions are defined and the test data is acquired with the help of finite element analysis results. To correlate material constants, modal test is required. Bending rigidity of material is optimized with the modal test as seen in Figure 1. With the correlated FEA model (natural frequencies are correlated only), structural damping and stiffness characterization of the connection elements can be modified in order to converge the test data.

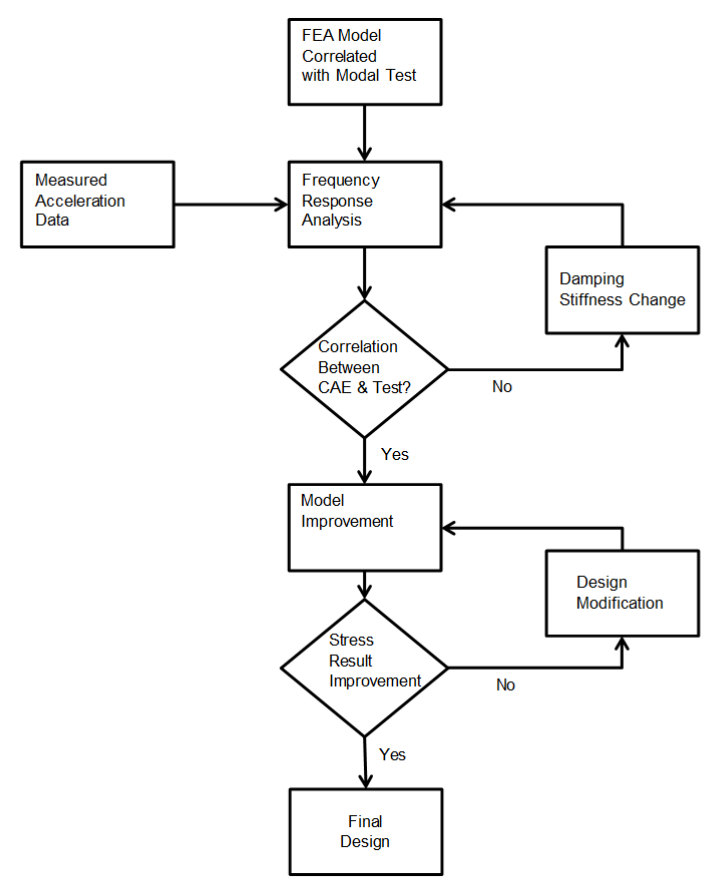

Fig. 9. Flowchart of Correlation Study

Fig. 7. Example of Laminated Steel 
By doing fast fourier transformation (FFT) the experimental data is converted to frequency scale and this data is used to perform the frequency response analysis. In the correlation part, RPM range where acceleration response is higher with respect to other RPM values, should be chosen for the study. By changing property of bushing element, nonstructural mass of oil and structural damping of laminated steel, best correlation is achieved. As shown in Figure 11 and Figure 12 the correlation between the experimental and CAE result is sustained.

The acceleration data from the block is the input part of the frequency response analysis. By using multiple excitation data, frequency response analysis of the oil pan is performed. In Figure 10, the example of this excitation data is given.

Correlation between accelerations on the oil pan and results of FEA analysis is crucial for the model optimization. The example of the correlated results is shown in Figure 11. Analysis revealed that at lower frequencies there is a good correlation between test data and analysis results. However, correlation is deteriorating because of the sloshing of the oil at higher frequencies. Other important item is stress correlation. RKU model is generally used for the dynamic analysis and stress results are not credible. To assure the stress results, also strain-gages are implemented on oil pan. Von-Misses stress results in Figure 12 which is derived from strain gage test data, show that RKU method gives rational stress results. After correlating FEA model, alternative geometry designs of the oil pan are studied for the optimization. Final design which satisfies the design target is achieved at the end of analysis loops. As a result all the steps mentioned in Figure 9 are completed.

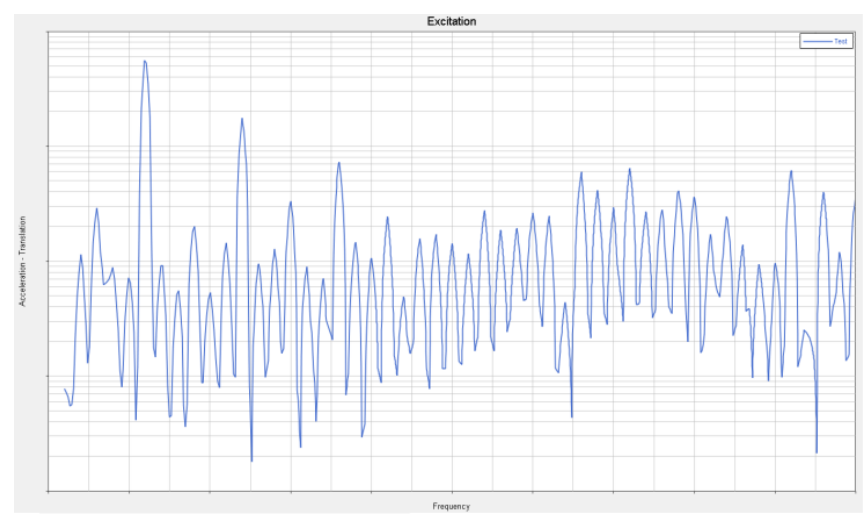

Fig. 10. Example of Excitation

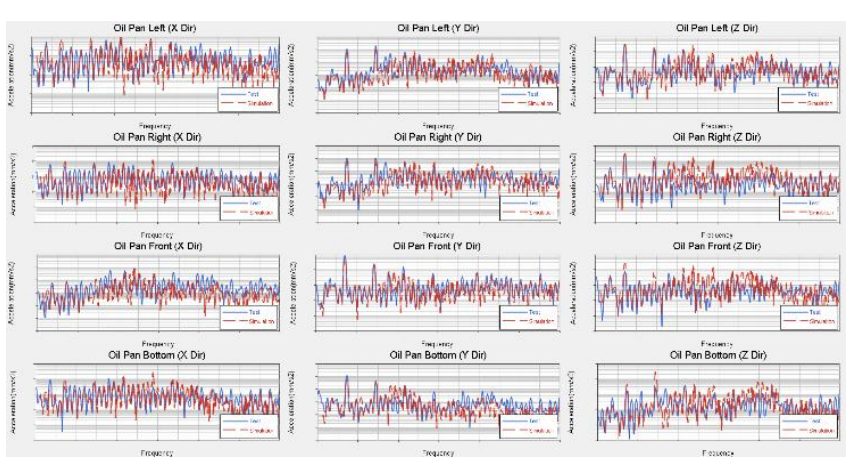

Fig. 11. Correlation Result and Test Result Comparison
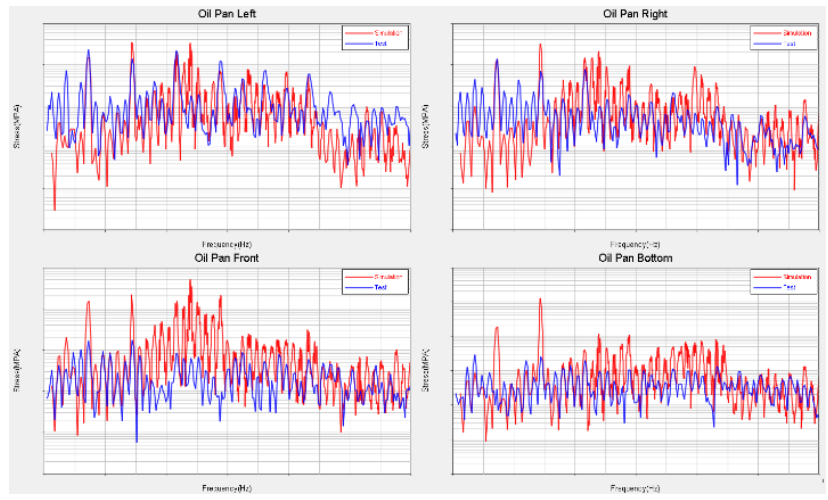

Fig. 12. Von Mises Stress Comparison (CAE Result vs Test Result)

\subsection{FEA Results}

At the end of study, it is observed that there is a good correlation at the side panels. However, at the bottom of the panel, correlation is not as good as side panels. This is the result of the higher fluid pressure at the bottom panel which is not simulated by adding non-structural mass. This situation is not critical since maximum Von-Misses stress is observed on the side panels. Also at the higher frequencies, correlation is becoming deteriorating because of the sloshing. However, maximum stress is not observed at higher frequencies which are seen both in the analysis result and the test data. As a result, the critical stress location at the first design is moved to the lower part of the side panel and magnitude of VonMisses stress is decreased to 55\% of the first design which is shown in Figure 13.

Acceleration value comparison for the primary and secondary design with $30 \mathrm{~L}$ and $50 \mathrm{~L}$ oil filling configurations is given in Figure 14. It can be clearly observed that 50L oil filling is more than 2 times critical than $30 \mathrm{~L}$. Also with the secondary oil pan design almost $60 \%$ of improvement is gained at both oil filling configurations.

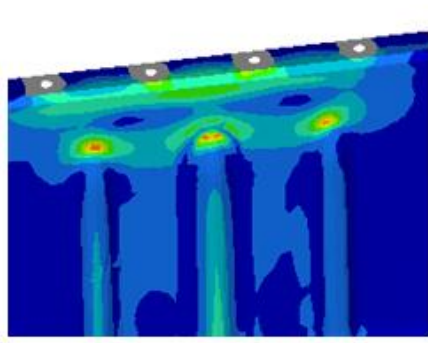

(a)

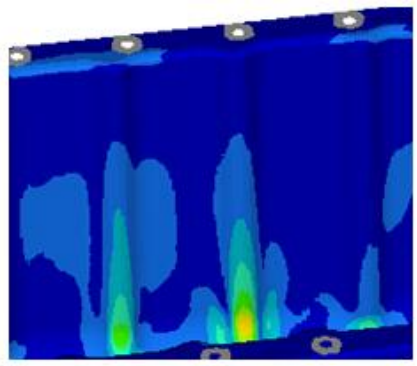

(b)
Fig. 13. Von-Mises Stress Location Preliminary Design (a) \& Final Design (b) 


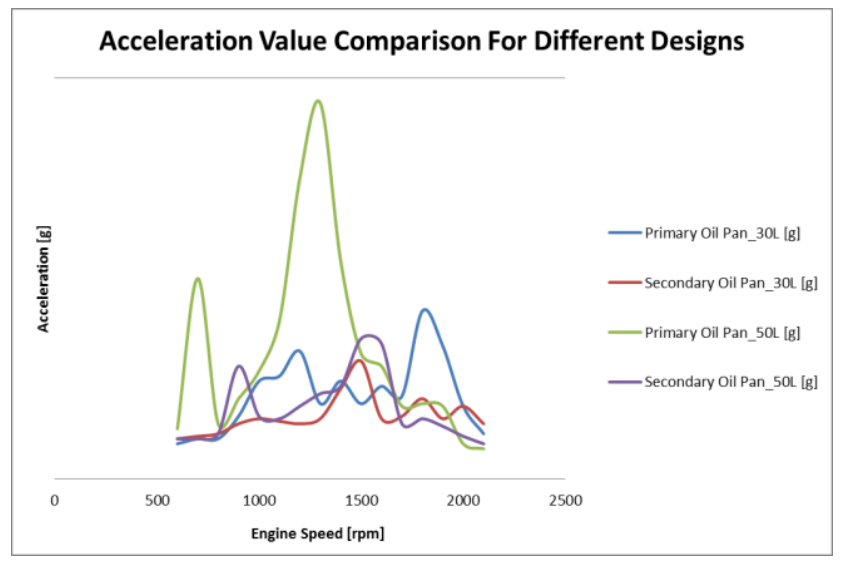

Fig. 14. Acceleration Value Comparison for Different Designs \& Oil Filling Configurations

\section{Conclusion}

In today's competitive production market using the right methodology and manufacturing, a fail-safe design at first trial is an important aspect that the research and development companies aim. But due to real life's unpredictable conditions, obtaining this fail-safe design is not always possible in virtual environment, especially on engines and engine components, manufactured with today's complex production methods and working as a thermomechanical end product. Therefore, simultaneous design and development works, integrating $\mathrm{CAE}$ analysis with experimental measurement techniques is used more effectively resulting with a single loop design. In this paper, the main steps and advantages of this kind of an approach is explained on a diesel engine oil pan.

First, a primary 3D dummy design is generated and a production method and material intent sample is produced accordingly. Using the modal shape and frequency data gained from the hammer test with the oil filled sump, CAE modal analysis is generated and the first challenge of modelling the liquid is overcome.

Secondly, from the predetermined critical stress locations obtained from CAE durability analysis, the block is instrumented with four accelerometers and the oil pan is instrumented with 4 strain gages and 4 accelerometers. Two configurations of oil filling, $30 \mathrm{~L}$ and $50 \mathrm{~L}$, are tested. With the refined CAE durability analysis, the second challenge of material nonlinearity and thinning is also overcome. According to the acceleration data the 50L oil filling found more than 2 times critical compared to 30L. Also in terms of max. principle stress, 50L oil filling found almost 6 times critical compared to $30 \mathrm{~L}$ especially at the side panels of the oil pan.

Lastly, with the CAE durability simulation, a secondary 3D design proposal is established, a sample is produced, instrumented again with accelerometers \& gages and tested. Almost 55\% improvement is gained in terms of Von Misses stresses and the critical stress locations at the first design is moved to the lower part of the side panel with much lower magnitudes. Hence, a method to perform a fail-safe oil pan design in single loop is verified.

\section{Acknowledgements}

The authors are indebted to the Ford Otosan Gölcük Engine Dynamometer Laboratory for the support required to complete project. Special thanks are given to Dr. Serdar AKCA for his support and encouragement throughout this project.

\section{References}

[1] Anab Akanda, Gregory M. Goetchius, "Representation of Constrained/Unconstrained Layer Damping Treatments in FEA/SEA Vehicle System Models: A Simplified Approach", SAE, 1999.

[2] Peter Andren, Tommy Falk, Juha Plunt "Analytical and Finite Element Design of Structural Damping using a Parametric Formulation for the Damping Material Properties", Internoise, 1999.

[3] Sean Bennett, "Modern Diesel Technology: Diesel Engines 2nd Edition", 2014.

[4] Sean Bennett "Medium/Heavy Duty Truck Engines, Fuel \& Computerized Management Systems, 4th Edition", 2013.

[5] Barry Kluczyk, "High-Performance New Hemi Builder's Guide: 2003-Present”, SA Design, 2007.

[6] Sean Bennett, "Modern Diesel Technology: Light Duty Diesels", 2012.

[7] "Heat Treating, Including Steel Heat Treating In the New Millennium: An International Symposium in Honor of Professor George Krauss", Sandra J. Midea, George D Pfaffmann, ASM International, 2000.

[8] "Deep Drawing: Benefits and Industrial Applications", http://www.thomasnet.com/articles/custommanufacturing-fabricating/deep-drawing.

[9] MSC Nastran User Manual. 